\title{
Estudo da prevalência da diabetes e das suas complicações numa coorte de diabéticos portugueses: um estudo na Rede Médicos-Sentinela
}

Isabel Marinho Falcão, ${ }^{*}$ Cristina Pinto, ${ }^{* *}$ Joana Santos, ${ }^{* * *}$ Maria de Lourdes Fernandes, ${ }^{* * * *}$ Leonor Ramalho, $* * * * *$ Eleonora Paixão, $* * * * * *$ José Marinho Falcão*******

\section{RESUMO}

Objectivos: Caracterizar uma coorte de diabéticos em relação ao sexo e idade; estimar a prevalência de diabetes de tipo 1 e 2; caracterizar os diabéticos de tipo 2 em relação à terapêutica prescrita, hábitos tabágicos e alcoólicos, ida à consulta de oftalmologia e a alguns dados bioquímicos e biométricos; estimar a prevalência das complicações da diabetes de tipo 2 .

Tipo de Estudo: Fase transversal, com a duração de 1 ano, de um estudo de follow up com a duração de 3 anos.

Local: Utentes de vários Centros de Saúde.

População: Utentes diabéticos inscritos nas listas de 66 médicos de família que colaboram com a Rede Médicos-Sentinela, num total de 4.583.

Métodos: Inquérito feito aos médicos sobre os utentes diabéticos inscritos nas respectivas listas. Os dados foram analisados no Departamento de Epidemiologia do Instituto Nacional de Saúde Dr. Ricardo Jorge.

Resultados e Conclusões: A taxa de prevalência de diabetes de tipo 1 foi estimada em 0,1\% e a de tipo 2 em 5,4\%. A terapêutica mais frequente associada à diabetes foi a da hipertensão, em 3.265 (75,5\%) casos. A interpretação dos resultados relacionados com as complicações da diabetes deve ser cuidadosa, uma vez que o número de casos é reduzido. Identificaram-se complicações da diabetes em 816 (18,7\%) diabéticos de tipo 2, sendo a retinopatia diabética a mais frequente, em $356(11,4 \%)$ casos. Uma vez que apenas cerca de metade $(1.790 ; 48,7 \%)$ dos diabéticos tinha ido à consulta de oftalmologia no ano anterior, poder-se-á estar a subestimar a prevalência desta complicação.

Apesar do grande volume de dados, sabe-se que vários factores poderão ter enviesado os resultados, pelo que deverão ser considerados como valores mínimos.

Palavras-chave: Diabetes; Prevalência; Complicações; Terapêutica; Rede Médicos-Sentinela.

\footnotetext{
*Médica de Família, Direcção-Geral da Saúde

**Médica de Família do CS Sta. Cruz do Bispo

***Médica de Família do CS Senhora da Hora

****Médica de Família do CS Oeiras

*****Médica de Família do CS da Venda-Nova

******Estatista do Instituto Nacional de Saúde Dr. Ricardo Jorge

*******Director do Departamento de Epidemiologia do Instituto Nacional

de Saúde Dr. Ricardo Jorge
}

\section{INTRODUÇÃO}

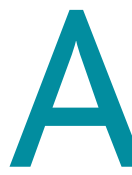

Diabetes Mellitus é uma doença crónica, frequente, que requer tratamento médico contínuo e ensino de auto-cuidados ao doente.

Trata-se duma doença com consequências importantes ao nível da saúde global e da qualidade de vida dos pacientes e das suas famílias. As primeiras re- 
ferências a esta doença remontam a 1550 anos A.C, no papiro de Ebers, descoberto no Egipto, onde era, então, tida como doença rara. ${ }^{2}$

A diabetes é considerada, por alguns autores, como uma epidemia global. Em 2003 estimou-se que cerca de 194 milhões de pessoas, em todo o mundo, tinham diabetes, sendo 5,1\%, no grupo etário 20-79 anos. Esta estimativa previa um aumento para cerca de 333 milhões, até 2025, sendo 6,3\% na população adulta. Prevê-se que o maior aumento absoluto e proporcional de casos irá ocorrer nos países em desenvolvimento, onde se espera que a prevalência aumente de $4,2 \%$ para $5,6 \%$. As regiões Europeia e do Pacífico Ocidental têm actualmente o maior número de diabéticos. No entanto, a taxa de prevalência de 3,1\%, na região do Pacífico Ocidental, é significativamente menor do que os 7,9\% da região da América do Norte e os 7,8\% da região Europeia. Cerca de metade de todos os recursos económicos dispendidos no tratamento da diabetes é utilizada no tratamento das suas complicações. A tendência crescente da prevalência da diabetes irá levar a um encargo financeiro cada vez maior, a não ser que sejam tomadas medidas para prevenir o seu aparecimento bem como o das respectivas complicações. $^{3}$

$\mathrm{O}$ aumento dos factores de risco para a diabetes de tipo 2, tais como a obesidade ${ }^{4,5}$ e a falta de actividade física adequada, ${ }^{6}$ têm contribuído para o aumento da diabetes no mundo inteiro; e o envelhecimento da população, a nível mundial, está também a contribuir para o aumento da prevalência desta doença. ${ }^{7}$

Segundo a Organização Mundial da Saúde, estima-se que, em todo o mundo, haja mais de 180 milhões de diabéticos, sendo provável que esse número exceda a sua duplicação em 2030. Estimou-se que, em 2005, cerca de 1,1 milhão de pessoas terá morrido por diabetes; e que quase $80 \%$ das mortes por diabetes terão ocorrido em países menos desenvolvidos. A estratégia da OMS para combater o provável aumento da prevalência da diabetes, nos próximos anos, baseia-se na dieta alimentar, para a obtenção de um peso corporal saudável; e a sua consequente manutenção, através do aumento da actividade física, com a execução de $30 \mathrm{minu}$ tos, pelo menos, de actividade física regular, de intensidade moderada, na maior parte dos dias. Em quase todas as sociedades desenvolvidas, a diabetes está entre as principais causas de cegueira, de insuficiência renal e de amputação dos membros inferiores; e é ainda uma das principais causas de morte, pois cerca de $70-80 \%$ dos diabéticos morrem de doença cardiovascular. ${ }^{8}$

A diabetes é uma doença que acarreta enorme sofrimento humano, bem como elevados custos sócio-económicos originados por uma morbilidade e mortalidade prematuras. ${ }^{9}$

Em Portugal, nas últimas décadas, a diabetes tem tido uma atenção crescente por parte dos técnicos e das instituições de saúde. De facto, o primeiro passo foi dado em 1983, com a criação da Carreira de Clínica Geral, que permitiu à população portuguesa a sua inscrição em médicos de família, facilitando assim a identificação dos diabéticos; e depois, em 1989, quando foi assinada a Declaração de St. Vincent, ${ }^{10}$ reconhecendo a diabetes como um grave e crescente problema de saúde pública e comprometendo-se Portugal a criar condições que permitissem reduzir significativamente o peso da doença. Em 1992 surgiu então o Programa Nacional de Controlo de Diabetes Mellitus, criado pela Direcção-Geral dos Cuidados de Saúde Primários, que, em 1998, sofreu um novo impulso ao ser-lhe reconhecida a necessidade de revisão. O cumprimento das estratégias definidas no Plano Nacional de Saúde 2004/2010 motivaram uma nova revisão do Programa, que, em 11 de Novembro de 2007, foi objecto duma circular normativa da Direcção-Geral da Saúde, em vigor até hoje. $^{11}$

Apesar de todos estes passos, dados no sentido de melhorar o conhecimento sobre a epidemiologia da diabetes e o seu controlo e retardando, tanto quanto possível, o aparecimento das complicações associadas à doença, muito se tem discutido sobre qual será, de facto, a prevalência da diabetes em Portugal. Num estudo realizado e publicado pela Rede Médicos-Sentinela no início dos anos 90, foi estimada uma prevalência de $2,0 \%$, que se sabe estar sub-avaliada; ${ }^{12}$ segundo o $4^{\circ}$ Inquérito Nacional de Saúde realizado em 2005-2006, foi estimada em $6,5 \%$ a percentagem da população que declarou ter diabetes, o que significa que, a ser verdade, existiriam cerca de 650.000 diabéticos em Portugal;13 ora, segundo a Associação Portuguesa de Diabéticos de Portugal, calcula-se que em Portugal existam entre 400 a 500 mil diabéticos; ${ }^{14}$ e, de acordo com estimativas da Associação Americana de Diabetes, em Portugal, em 2007 existiriam cerca de 900 mil diabéticos. ${ }^{15}$ 
Perante estimativas tão diferentes, a Rede Médicos-Sentinela decidiu repetir, de 2005 a 2007, o estudo sobre «Prevalência da diabetes e sua incidência numa coorte de diabéticos portugueses».

Neste artigo serão apenas descritos alguns resultados da $1^{\text {a }}$ fase desse estudo (2005), com os seguintes objectivos:

- Caracterizar a coorte de diabéticos em relação ao sexo e idade;

- Estimar a prevalência de diabetes de tipo 1 e 2;

- Caracterizar os diabéticos de tipo 2 em relação à terapêutica prescrita, hábitos tabágicos e alcoólicos, ida à consulta de oftalmologia e dados bioquímicos e biométricos;

- Estimar a prevalência de complicações da diabetes de tipo 2.

\section{MÉTODOS}

Neste artigo serão apenas descritos alguns dos resultados obtidos na $1^{\text {a }}$ fase do estudo de follow up «Prevalência da diabetes e incidência das suas complicações numa coorte de diabéticos portugueses», realizado no âmbito da actividade da Rede Médicos-Sentinela.

Médicos-Sentinela é um sistema de informação em saúde constituído por cerca de uma centena e meia de médicos de família que, trabalhando em Centros de Saúde do território do Continente e das Regiões Autónomas, participam, de forma voluntária, no estudo de várias doenças ou de situações relacionadas com saúde. A coordenação da actividade desta Rede é feita no Departamento de Epidemiologia do Instituto Nacional de Saúde Dr. Ricardo Jorge (INSA), designado até Agosto de 2007 por Observatório Nacional de Saúde (ONSA).

Cada médico participante tem a seu cargo uma população de utentes inscrita no Centro de Saúde, cuja composição é conhecida em relação ao sexo e ao grupo etário. Esta informação é actualizada anualmente. A base populacional de Médicos-Sentinela permite calcular estimativas de incidência de várias doenças, bem como estimar índices de frequência de situações relacionadas com saúde, de exames complementares de diagnóstico ou de prescrição de medicamentos; e o seguimento dos utentes e das respectivas famílias, ao longo do tempo, que caracteriza a principal actividade do médico de família, facilita a realização de estudos de follow-up, que, pela natureza do seu delineamento, são habitualmente onerosos e de difícil execução.

Assim, aproveitando essa facilidade, foi pedido aos Médicos-Sentinela (MS) que, em 3 anos consecutivos (2005-2007), enviassem para o Departamento de Epidemiologia do INSA, através de suportes de notação criados para o efeito (em anexo), um conjunto de dados referentes aos utentes diabéticos inscritos nas respectivas listas. Foram 66 os médicos que declararam expressamente querer participar neste estudo. Os questionários não foram sujeitos a nenhum estudo de validação, dada a natureza do sistema Médicos-Sentinela, mas foram construídos com base na experiência adquirida no estudo semelhante, realizado no âmbito da Rede Médicos-Sentinela de 1993 a 1995, também sobre diabetes.

O estudo decorreu de 01 de Janeiro de 2005 a 31 de Dezembro de 2007, e incluiu três observações de utentes diabéticos, sendo a primeira em 2005 (fase transversal do estudo), a segunda em 2006 e a terceira em 2007.

$\mathrm{Na} 1^{\text {a }}$ fase, transversal, deste estudo, foi seleccionada a coorte de diabéticos a estudar. Todos os utentes inscritos nas listas dos Médicos-Sentinela que concordaram em participar no estudo e aos quais tinha sido feito o diagnóstico de diabetes até 01/01/05 foram identificados e seleccionados.

A cada médico foi enviado, por correio postal, um conjunto de questionários (anexo no final) (tantos quantos os utentes diabéticos que o médico declarou estarem inscritos na sua lista), que depois de preenchidos foram enviados para o ONSA, também por correio postal. Os médicos que preferiram utilizar a INTERNET para o envio da informação, fizeram-no através da plataforma RIOS (www.insa-rios.net) onde é feita, parcialmente, a gestão da Rede Médicos-Sentinela e onde foram disponibilizados os referidos questionários acedidos através de password personalizada.

O questionário foi composto pelas seguintes variáveis: sexo e data de nascimento do diabético, número de anos de escolaridade com aproveitamento, ano de diagnóstico e tipo da diabetes, terapêutica que o utente fazia, alguns valores de indicadores bioquímicos (HbAlc, colesterol total, colesterol-HDL, colesterol-LDL, triglicéridos, creatinina, proteinúria, microalbuminúria) e biométricos (peso, altura e tensão arterial), hábitos tabágicos e alcoólicos, existência prévia de observação por oftalomologista e de complicações de diabetes (re- 
tinopatia diabética, diminuição grave da acuidade visual não corrigível, cegueira total, necessidade de hemodiálise, realização de transplante renal, antecedentes de acidente vascular cerebral, de enfarte agudo do miocárdio, de bypass arterial periférico, de angioplastia ou bypass coronário e de amputação de membros).

O critério de diagnóstico de diabetes de tipo 1 e 2 não foi previamente definido, tendo sido aceite a informação enviada pelo médico.

Para classificar os hábitos alcoólicos foram recomendados os seguintes critérios da OMS: ${ }^{16}$ foi considerado bebedor excessivo o diabético do sexo masculino que ingeria 30 ou mais gramas de álcool por dia; e para as mulheres o valor foi de 15 ou mais gramas de álcool/dia. Estes valores traduzem-se nas seguintes equivalências: 30 gramas $=3 \mathrm{dl}$ de vinho ( $\sim 2$ copos de vinho) ou 2 cervejas de 3,3 dl; 15 gramas=1,5 dl de vinho $(\sim 1$ copo de vinho) ou uma cerveja de 3,3 dl. Nos indivíduos não diabéticos considera-se bebedor excessivo qualquer indivíduo que ingira 50 ou mais gramas de álcool por dia (5 dl de vinho); nas mulheres 40 ou mais gramas de álcool por dia (4 dl de vinho); ou então quando se verificar o consumo de 5 ou mais bebidas de ocasião durante $o$ fim-de-semana.

O valor de Colesterol-LDL foi recalculado, no Departamento de Epidemiologia do INSA, através da fórmula de Friedewald ${ }^{17}$ que permite o seu cálculo de forma indirecta:

$$
\mathrm{LDL}=\mathrm{CT}-\mathrm{HDL}-\mathrm{TG} / 5
$$

Uma vez que não foram encontrados valores acima de $400 \mathrm{mg} / \mathrm{dl}$, não foi necessário utilizar os valores dados pelo método enzimático.

Para o cálculo do Índice de Massa Corporal (IMC) foi utilizada a fórmula: ${ }^{18}$

\section{IMC=Peso/Altura2}

Consideram-se como complicações da diabetes as seguintes: retinopatia diabética; acidente vascular cerebral; enfarte agudo do miocárdio; cegueira total; diminuição grave da acuidade visual, não corrigível $(<1 / 10$ no melhor olho) e amputações.

Consideraram-se também como complicações da diabetes as seguintes intervenções terapêuticas (feitas com o objectivo de resolver complicações directas da diabetes): angioplastia ou bypass coronário; bypass arterial periférico; hemodiálise e transplante renal.

Na primeira fase, transversal, deste estudo, os dados foram enviados para o Departamento de Epidemiologia do INSA, até ao final do ano de 2005. Os que chegaram por correio postal foram introduzidos em computador tendo sido criada uma base única final, à qual se juntaram os dados enviados por via telemática. Esta base final foi então validada, tendo sido questionados os médicos sobre todos os valores duvidosos ou impossíveis que foram encontrados e corrigidos posteriormente.

\section{POPULAÇÃO SOB OBSERVAÇÃO}

Foram incluídos todos os utentes que integravam, em 2005, as listas dos médicos participantes no estudo, tendo sido considerada a respectiva distribuição por sexo e grupo etário. Assim, a taxa de prevalência da diabetes de tipo 1 e 2 foi estimada utilizando como denominador a população inscrita, em 1 de Janeiro de 2005, nas listas dos médicos participantes no estudo, num total de 111.152 utentes.

Para ajustar as taxas de prevalência à população portuguesa foi utilizada a estimativa da população portuguesa residente em $31 / 12 / 2006$, i.e, a mais recente disponível na data de análise dos dados. ${ }^{19}$

Para os vários indicadores bioquímicos estudados foram definidos valores máximos e mínimos possíveis, tendo em conta os valores de referência do laboratório do INSA, a linearidade de cada método de doseamento utilizado e os valores descritos na literatura; sempre que se verificou ausência de informação publicada sobre os valores normais ou os limites possíveis dos parâmetros em estudo, foram considerados como valores limite os máximos e mínimos identificados e confirmados por cada MS, após uma validação adicional por telefone.

Em relação à microalbuminúria foram encontrados resultados duvidosos que não foi possível confirmar, pelo que esses dados não serão tratados neste relatório.

Assim, foram considerados como possíveis os seguintes valores:

- HbAlc: $>=2,5$ e $<=16,7 \%$

- Colesterol total: $>=100 \mathrm{e}<=1.000 \mathrm{mg} / \mathrm{dl}$

- Colesterol-HDL: $>=23 \mathrm{e}<=110 \mathrm{mg} / \mathrm{dl}$

- Colesterol-LDL: $>=38$ e > =320 mg/dl

- Triglicéridos: > $\quad>26 \mathrm{e}<=4.851 \mathrm{mg} / \mathrm{dl}$

- Creatinina: $\quad>=0,10$ e $<=12,90 \mathrm{mg} / \mathrm{dl}$

- Proteinúria: tem/não tem 


\section{OBJECTIVOS DO TRATAMENTO, SEGUNDO A APMCG}

A análise dos dados bioquímicos e biométricos teve em conta as Recomendações de 2006 do Grupo de Estudos da Diabetes da APMCG na diabetes tipo 2 para a prática clínica diária em Cuidados de Saúde Primários. ${ }^{20}$

Foi, assim, estimada a proporção de diabéticos que cumpriam aqueles objectivos na data da observação, apesar de este não ser um dos objectivos iniciais do estudo.

O tratamento dos dados foi feito com o programa estatístico SPSS (Statistical Package for Social Sciences) versão $15 .{ }^{21}$

A análise de variáveis qualitativas (categoriais) foi realizada principalmente através de contagens e frequências relativas (percentagens). A análise de variáveis quantitativas (contínuas) foi realizada através de estatísticas como a média, mediana, mínimo, máximo e desvio padrão.

As variáveis consideradas relevantes foram desagregadas por sexo e idade.

Para testar a associação (ou independência) entre variáveis categoriais, utilizou-se o teste de Qui-Quadrado de Pearson ou, como alternativa, em caso de tabelas de contingência $2 \times 2$, o teste Exacto de Fisher.

Para testar a existência de diferenças entre parâmetros de tendência central, em variáveis quantitativas, utilizou-se o teste não paramétrico de Mann-Whitney, como alternativa ao teste $t$ de Student.

Foi estabelecido em $5 \%$ o nível de significância dos testes, rejeitando-se a hipótese nula quando a probabilidade de significância do teste ( $p$-value) foi inferior a este valor.

Dada a elevada prevalência da diabetes de tipo $2 \mathrm{em}$ relação à de tipo 1, este relatório incidirá, detalhadamente, apenas sobre a primeira.

\section{RESULTADOS}

Médicos participantes e $\mathrm{n}^{\circ}$ de casos seleccionados Participaram, na fase transversal do estudo, 66 médicos de família da Rede Médicos-Sentinela (48,2\% do total de médicos da Rede).

Esses médicos enviaram dados referentes a 4.583 utentes diabéticos inscritos nas respectivas listas, cujo diagnóstico de diabetes tinha sido feito até 01/01/05.

O número mínimo e máximo de casos enviados por cada médico foi, respectivamente, 10 e 157, sendo a média de 69,4, a moda 39 e a mediana 65 .

\section{Caracterização da coorte de diabéticos por sexo e grupo etário}

Verificou-se que dos 4.561 diabéticos (99,5\% do total) em que havia dados sobre o sexo, $47,0 \%$ (2.142) eram do sexo masculino e $53,0 \%$ (2.419) do feminino. Em relação à idade, 32,7\% (1.497) dos diabéticos pertenciam ao grupo etário dos 65-74 anos (Quadro I).

\section{Estimativa de prevalência da diabetes \\ Diabetes de tipo 1}

O número de casos reportados de diabetes de tipo 1 foi 158 sendo $51,3 \%$ (79) referentes a homens, $48,7 \%$ (75) a mulheres e $2,5 \%$ (4) sem informação sobre o sexo.

A taxa de prevalência de diabetes de tipo 1 foi estimada em $0,1 \%$ para ambos os sexos.

\section{Ajustamento à população portuguesa residente}

Ajustando as estimativas de prevalência da diabetes de tipo 1 à população residente ${ }^{19}$ obteve-se um total de 15.003 casos, sendo 7.837 do sexo masculino e 7.166 do sexo feminino.

\begin{tabular}{|c|c|c|c|}
\hline \multicolumn{4}{|c|}{$\begin{array}{l}\text { QUADRO I. Caracterização da coorte de diabéticos } \\
\text { por sexo e grupo etário }\end{array}$} \\
\hline Sexo & $\mathbf{n}$ & $\%$ & $\%$ sem inf. \\
\hline Total & 4.561 & & 0,5 \\
\hline Masculino & & $47,0(2.142)$ & \\
\hline Feminino & & $53,0(2.419)$ & \\
\hline Grupo etário & $\mathrm{n}$ & $\%$ & \\
\hline Total & 4.575 & & \\
\hline $00-09$ & & $0,0(1)$ & \\
\hline $10-14$ & & $0,1(6)$ & \\
\hline $15-24$ & & $0,5(21)$ & \\
\hline $25-34$ & & $1,1(50)$ & \\
\hline $35-44$ & & $4,1(186)$ & \\
\hline $45-54$ & & $13,6(624)$ & \\
\hline $55-64$ & & 24,3 (1.114) & \\
\hline $65-74$ & & $32,7(1.497)$ & \\
\hline 75 e + & & $23,5(1.076)$ & \\
\hline
\end{tabular}

(..) nº de indivíduos com resposta válida 


\begin{tabular}{|c|c|c|c|}
\hline \multicolumn{4}{|c|}{$\begin{array}{l}\text { QUADRO II. Estimativa da taxa de prevalência (\%) dos } \\
\text { diabéticos de tipo 2, por sexo e grupo etário }\end{array}$} \\
\hline Grupo etário & Homens & Mulheres & $\mathrm{H}+\mathrm{M}$ \\
\hline $25-34$ & $\begin{array}{c}0,1 \\
(11)\end{array}$ & $\begin{array}{l}0,1 \\
(7)\end{array}$ & $\begin{array}{c}0,1 \\
(18)\end{array}$ \\
\hline $35-44$ & $\begin{array}{r}1,0 \\
(80)\end{array}$ & $\begin{array}{c}0,8 \\
(65)\end{array}$ & $\begin{array}{c}0,9 \\
(145)\end{array}$ \\
\hline $45-54$ & $\begin{array}{c}4,7 \\
(323)\end{array}$ & $\begin{array}{c}3,7 \\
(269)\end{array}$ & $\begin{array}{c}4,2 \\
(592)\end{array}$ \\
\hline $55-64$ & $\begin{array}{c}9,7 \\
(531)\end{array}$ & $\begin{array}{c}8,6 \\
(544)\end{array}$ & $\begin{array}{c}9,1 \\
(1.075)\end{array}$ \\
\hline $65-74$ & $\begin{array}{r}13,6 \\
(667)\end{array}$ & $\begin{array}{c}12,8 \\
(785)\end{array}$ & $\begin{array}{c}13,1 \\
(1.452)\end{array}$ \\
\hline 75 e + & $\begin{array}{c}11,9 \\
(418)\end{array}$ & $\begin{array}{c}11,5 \\
(631)\end{array}$ & $\begin{array}{c}11,6 \\
(1.049)\end{array}$ \\
\hline Total & $\begin{array}{c}5,4 \\
(2.030)\end{array}$ & $\begin{array}{c}5,4 \\
(2.301)\end{array}$ & $\begin{array}{c}5,4 \\
(4.331)\end{array}$ \\
\hline
\end{tabular}

(..) nº de indivíduos com resposta válida; sem informação: 0,6\% (25)

\section{Diabetes de tipo 2}

Considerando os diabéticos com 25 e mais anos, uma vez que não havia diabéticos de tipo 1 com menos de 25 anos de idade, a prevalência da diabetes de tipo 2 foi estimada em 5,4\%, igual para ambos os sexos (Quadro II).

\section{Ajustamento à população portuguesa residente}

Ajustando as estimativas de prevalência da diabetes de tipo 2 à população residente ${ }^{19}$ obtivemos os resultados expressos no Quadro III.

Pode, assim, dizer-se que, em 31/12/06, existiriam em Portugal, na população residente com 25 e mais anos, 414.214 diabéticos de tipo 2.

Verificou-se que, em ambos os sexos, nos diabéticos com menos de 25 anos de idade só foram identificadas diabetes de tipo 1; pelo contrário, nos diabéticos com 25 e mais anos foram identificadas diabetes de tipo 2 , gestacional e outro tipo. Saliente-se que a percentagem de casos de cada um dos tipos de diabetes foi semelhante nos homens e nas mulheres.

\section{Distribuição percentual do tipo de diabetes}

Verificou-se que 95,3\% dos diabéticos eram de tipo 2 e $3,5 \%$ do tipo 1 . Os restantes $1,2 \%$ eram de «outro tipo» ou «gestacionais» (Quadro IV).
QUADRO III. Estimativa do número de casos de diabetes de tipo 2 na população portuguesa residente, por sexo e grupo etário

\begin{tabular}{l|c|c|c} 
Grupo etário & Homens & Mulheres & \multicolumn{1}{c}{$\mathbf{H + M}$} \\
\hline $25-34$ & 826 & 814 & 1.640 \\
\hline $35-44$ & 7.813 & 6.360 & 14.173 \\
\hline $45-54$ & 32.814 & 27.196 & 60.010 \\
\hline $55-64$ & 55.754 & 55.283 & 111.037 \\
\hline $65-74$ & 60.396 & 71.357 & 131.753 \\
\hline 75 e + & 37.251 & 58.350 & 95.601 \\
\hline Total (25 e + anos) & 194.855 & 219.359 & 414.214 \\
\hline
\end{tabular}

\section{Diabetes de tipo 2}

Uma vez que os utentes com idade inferior a 25 anos eram todos diabéticos de tipo 1, consideraram-se, a partir daqui para a análise, apenas os diabéticos de tipo 2 com 25 e mais anos, num total de 4.356 .

\section{Terapêuticas prescritas}

Foi possível estudar a terapêutica prescrita a 4.327 diabéticos (99,3\% da coorte).

Verificou-se que $3.781(87,4 \%)$ diabéticos de tipo 2 faziam terapêutica com antidiabéticos orais e 404 (9,3\%) com insulina. A terapêutica com antihipertensores foi referida em $3.265(75,5 \%)$ casos e a dieta/exercício físico em 2.717 (62,8\%). Os antidislipidémicos tinham sido prescritos a $2.437(56,3 \%)$ utentes, a terapêutica anti-trombótica em 1.606 (37,1\%) casos e a terapêutica para prevenção/tratamento de nefropatia em 1.300 (30,0\%) (Quadro V).

\section{Hábitos tabágicos}

Verificou-se que 3.903 diabéticos (93,0\%) não tinham

\section{QUADRO IV. Distribuição percentual do tipo de diabetes}

\begin{tabular}{l|c} 
Tipo de diabetes & $\%$ \\
\hline Tipo 1 & $3,5(158)$ \\
\hline Tipo 2 & $95,3(4.356)$ \\
\hline Gestacional & $0,3(13)$ \\
\hline Outro tipo & $0,9(42)$ \\
\hline Total & $100,0(4.569)$
\end{tabular}

(..) n de indivíduos com resposta válida; sem informação: 0,3\% (14) 


\section{QUADRO V. Terapêuticas prescritas aos diabéticos de} tipo $2(\mathrm{~N}=4.327)$

\begin{tabular}{l|c} 
Terapêutica* & $\%$ \\
\hline Antidiabéticos orais & $87,4(3.781)$ \\
\hline Antihipertensores & $75,5(3.265)$ \\
\hline Dieta/exercício físico & $62,8(2.717)$ \\
\hline Antidislipidémicos & $56,3(2.437)$ \\
\hline Insulina & $9,3(4.04)$ \\
\hline Antitrombótica & $37,1(1.606)$ \\
\hline Prevenção/tratamento de nefropatia & $30,0(1.300)$
\end{tabular}

(..) n de indivíduos com resposta válidas; Sem informação: 0,7\% (29); *uma ou mais terapêuticas são possíveis

\begin{tabular}{|c|c|c|c|c|}
\hline \multicolumn{5}{|c|}{$\begin{array}{l}\text { QUADRO VI. Distribuição dos hábitos tabágicos dos } \\
\text { diabéticos de tipo } 2 \text {, segundo o sexo }\end{array}$} \\
\hline $\begin{array}{l}\text { Hábitos } \\
\text { tabágicos }\end{array}$ & $\begin{array}{c}\text { Homens } \\
\%\end{array}$ & $\begin{array}{c}\text { Mulheres } \\
\%\end{array}$ & $\begin{array}{c}\mathrm{H}+\mathrm{M} \\
\%\end{array}$ & p \\
\hline & & & & $<0,001^{\mathrm{a}}$ \\
\hline Fuma todos os dias & $\begin{array}{c}9,6 \\
(185)\end{array}$ & $\begin{array}{c}1,2 \\
(27)\end{array}$ & $\begin{array}{c}5,1 \\
(212)\end{array}$ & \\
\hline $\begin{array}{l}\text { Fuma rara ou } \\
\text { esporadicamente }\end{array}$ & $\begin{array}{c}3,5 \\
(68)\end{array}$ & $\begin{array}{c}0,6 \\
(13)\end{array}$ & $\begin{array}{r}1,9 \\
(81)\end{array}$ & \\
\hline Não fuma & $\begin{array}{c}86,9 \\
(1.683)\end{array}$ & $\begin{array}{c}98,2 \\
(2.220)\end{array}$ & $\begin{array}{c}93,0 \\
(3.903)\end{array}$ & \\
\hline Total & $\begin{array}{c}100,0 \\
(1.936)\end{array}$ & $\begin{array}{c}100,0 \\
(2.260)\end{array}$ & $\begin{array}{c}100,0 \\
(4.196)\end{array}$ & \\
\hline
\end{tabular}

ateste de $\chi^{2}$ de Pearson; sem informação: 3,7\% (160)

hábitos tabágicos e que apenas $212(5,1 \%)$ fumavam diariamente. Registou-se uma diferença estatisticamente significativa $(\mathrm{p}<0,001)$ entre os sexos em relação aos hábitos tabágicos. Assim, a proporção de homens fumadores diários $(9,6 \%)$ foi maior do que a das mulheres $(1,2 \%)$ (Quadro VI).

Comparando os hábitos tabágicos dos diabéticos estudados com os da população portuguesa verificou-se que, nesta, a percentagem de fumadores foi estimada em $19,6 \%$ para o Continente, $20,3 \%$ para a RAM e $24 \%$ para a RAA, sendo para o sexo masculino, respectivamente, $28,7 \%, 31,5 \%$ e $36,4 \%$, e para o sexo feminino $11,2 \%, 10,6 \%$ e $11,9 \% .{ }^{13} \mathrm{Na}$ coorte de diabéticos estudada apenas 5,1\% dos indivíduos fumava regularmente, sendo $9,6 \%$ do sexo masculino e $1,2 \%$ do feminino.

\begin{tabular}{|c|c|c|c|c|}
\hline \multicolumn{5}{|c|}{$\begin{array}{l}\text { QUADRO VII. Distribuição dos hábitos alcoólicos } \\
\text { dos diabéticos de tipo } 2 \text {, segundo o sexo }\end{array}$} \\
\hline $\begin{array}{l}\text { Hábitos } \\
\text { alcoólicos }\end{array}$ & $\begin{array}{c}\text { Homens } \\
\%\end{array}$ & \begin{tabular}{|c|} 
Mulheres \\
$\%$
\end{tabular} & $\begin{array}{c}\mathrm{H}+\mathrm{M} \\
\%\end{array}$ & $P$ \\
\hline & & & & $<0,001^{a}$ \\
\hline Bebe excessivamente & $\begin{array}{l}11,9 \\
(228)\end{array}$ & $\begin{array}{c}1,2 \\
(27)\end{array}$ & $\begin{array}{c}6,1 \\
(255)\end{array}$ & \\
\hline $\begin{array}{l}\text { Bebe moderadamente } \\
\text { todos os dias }\end{array}$ & $\begin{array}{l}45,9 \\
(882)\end{array}$ & $\begin{array}{c}10,3 \\
(230)\end{array}$ & $\begin{array}{c}26,7 \\
(1112)\end{array}$ & \\
\hline $\begin{array}{l}\text { Bebe rara ou } \\
\text { esporadicamente }\end{array}$ & $\begin{array}{r}18,6 \\
(357)\end{array}$ & $\begin{array}{r}15,6 \\
(348)\end{array}$ & $\begin{array}{c}17,0 \\
(705)\end{array}$ & \\
\hline Não bebe & $\begin{array}{l}23,6 \\
(454)\end{array}$ & $\begin{array}{c}73,0 \\
(1.632)\end{array}$ & $\begin{array}{c}50,2 \\
(2.086)\end{array}$ & \\
\hline Total & $\begin{array}{c}100,0 \\
(1.921)\end{array}$ & $\begin{array}{c}100,0 \\
(2.237)\end{array}$ & $\left|\begin{array}{c}100,0 \\
(4.158)\end{array}\right|$ & \\
\hline
\end{tabular}

ateste de $\chi^{2}$ de Pearson; sem informação: sem informação: 4,5\% (198)

\section{Hábitos alcoólicos}

Verificou-se que 2.086 diabéticos $(50,2 \%)$ não tinham hábitos alcoólicos e que apenas 255 (6,1\%) bebiam excessivamente. Verificou-se haver diferença estatisticamente significativa $(p<0,001)$ entre os sexos em relação aos hábitos alcoólicos, tendo sido estimada em 11,9\% a proporção de homens que bebia excessivamente e em $1,2 \%$ a das mulheres (Quadro VII).

Comparando os hábitos alcoólicos dos diabéticos estudados com os da população portuguesa ${ }^{13}$ verificou-se que, nesta, a percentagem de indivíduos com hábitos alcoólicos foi estimada em 53,8\% para Portugal continental, $31,5 \%$ para a RAM e $48,8 \%$ para a RAA; em comparação nos diabéticos estudados obteve-se uma proporção de 49,8\% para Portugal continental e RAA.

\section{Consulta de oftalmologia}

Verificou-se que 1.790 diabéticos $(48,7 \%)$ foram à consulta de oftalmologia no ano de 2004, sendo 821 homens $(49,2 \%)$ e 969 mulheres (48,7\%). Não foi encontrada diferença estatisticamente significativa $(\mathrm{p}=0,643)$ entre os sexos em relação a esta variável.

\section{Dados bioquímicos e biométricos}

Os resultados obtidos serão descritos tendo em conta as Recomendações de 2006 do Grupo de Estudos da Diabetes da Associação Portuguesa dos Médicos de Clí- 
nica Geral (APMCG) na diabetes tipo 2 para a prática clínica diária em Cuidados de Saúde Primários. ${ }^{20}$

\section{HbA1C}

Verificou-se existir diferença estatisticamente significativa entre os valores médios de HbAlc no sexo masculino e feminino $(\mathrm{p}<0,001)$, sendo o valor médio de HbAlc superior nas mulheres $(7,34 \%)$ em relação ao dos homens $(7,03 \%)$.

Verificou-se que cerca de $52,2 \%$ dos diabéticos de tipo 2 cumpriam os objectivos de tratamento na data da observação, sendo os homens $55,4 \%$ e as mulheres $49,4 \%$. O grupo $25-34$ anos foi o que mais se aproximou dos objectivos de tratamento pretendidos uma vez que $80 \%$ dos diabéticos tinha valores de HbAlc $<7 \%$. Saliente-se que a percentagem de diabéticos que cumpriu os objectivos do tratamento foi maior nos homens do que nas mulheres, em todos os grupos etários.

Em ambos os sexos e no total de ambos os sexos foram encontradas diferenças estatisticamente significativas entre os grupos etários em relação à percentagem de diabéticos que atingiram os objectivos de tratamento expressos nas recomendações de 2006. Os grupos etários dos 35-44, 45-54 e dos 65-74 anos foram os que apresentaram diferenças significativas entre homens e mulheres $(\mathrm{p}=0,021 ; \mathrm{p}=0,021$ e $\mathrm{p}=0,023$ respectivamente).

\section{Colesterol total}

Verificou-se existir diferença estatisticamente significativa entre os sexos no que respeita aos valores médios de colesterol total $(\mathrm{p}<0,001)$, sendo o valor superior nas mulheres $(204,96 \mathrm{mg} / \mathrm{dl})$ em relação ao dos homens (196 mg/dl).

Verificou-se que $53 \%$ dos diabéticos de tipo 2 tinham valores de colesterol total abaixo dos valores recomendados como objectivo de tratamento, sendo $57,8 \%$ homens e 48,9\% mulheres. Foi no grupo etário 25-34 anos que os objectivos de tratamento foram mais bem cumpridos, pois $62,5 \%$ dos diabéticos tinha valores de colesterol total abaixo de $200 \mathrm{mg} / \mathrm{dl}$.

Verificou-se haver diferença estatisticamente significativa no sexo masculino, na distribuição da percentagem de diabéticos de tipo 2 que cumpriam os objectivos de tratamento em relação ao colesterol total, por grupo etário $(\mathrm{p}=0,010)$. Os grupos etários a partir dos 55 anos de idade apresentaram diferenças estatisticamente significativas entre homens e mulheres.

\section{Colesterol-HDL}

Verificou-se existir diferença estatisticamente significativa entre os sexos para os valores médios de colesterol-HDL ( $<<0,001)$, sendo o valor médio superior nas mulheres $(52,65 \mathrm{mg} / \mathrm{dl})$ em relação aos homens $(47,40$ $\mathrm{mg} / \mathrm{dl})$.

Em relação ao colesterol-HDL, apenas $43,6 \%$ dos diabéticos de tipo 2 tinha valores que cumpriam os objectivos de tratamento recomendados, sendo o grupo etário 25-34 anos o que revelou a proporção mais elevada de diabéticos nessas condições.

Verificaram-se diferenças estatisticamente significativas em todos os grupos etários, com excepção dos 25-34 anos, apresentado as mulheres as proporções mais elevadas de cumprimento dos objectivos propostos em relação à variável em estudo.

\section{Colesterol-LDL}

Verificou-se existir diferença estatisticamente significativa entre os sexos nos valores médios de colesterol-LDL $(\mathrm{p}=0,018)$, sendo o valor médio superior nas mulheres $(122,07 \mathrm{mg} / \mathrm{dl})$ em relação aos homens $(118,36 \mathrm{mg} / \mathrm{dl})$.

Verificou-se que apenas $29,3 \%$ dos diabéticos cumpriam os objectivos de tratamento recomendados, sendo o grupo 35-44 anos o que maior proporção de indivíduos incluiu nessas condições $(34,4 \%)$.

Verificou-se haver diferença estatisticamente significativa nas mulheres, em relação à distribuição dos valores de colesterol-LDL nos vários grupos etários $(\mathrm{p}=0,042)$; essas diferenças ocorreram nos grupos $35-44$ anos $(\mathrm{p}=0,004)$ e $75-79$ anos $(\mathrm{p}=0,037)$

\section{Triglicéridos}

Não foi encontrada diferença estatisticamente significativa entre os sexos para os valores médios de triglicéridos ( $\mathrm{p}=0,103)$.

Verificou-se que $60,2 \%$ dos diabéticos de tipo 2 tinham valores de triglicéridos abaixo dos recomendados como objectivo de tratamento, sendo $60,9 \%$ homens e $59,5 \%$ mulheres. Foi no grupo etário 80 e + anos que os objectivos de tratamento foram mais bem cumpridos, pois $65,3 \%$ dos diabéticos tinha valores de colesterol total abaixo de $200 \mathrm{mg} / \mathrm{dl}$. 
Verificou-se haver diferenças estatisticamente significativas nos homens e no total de ambos os sexos na distribuição da percentagem de diabéticos de tipo 2 que cumpriam os objectivos de tratamento em relação aos triglicéridos, por grupo etário $(\mathrm{p}<0,001)$. Os grupos etários a partir dos 75 anos de idade apresentaram diferenças estatisticamente significativas.

\section{Creatinina}

Verificou-se existir diferença estatisticamente significativa entre os sexos nos valores médios de creatinina $(\mathrm{p}<0,001)$, sendo o valor médio superior nos homens (1,00 mg/dl).

\section{Índice de Massa Corporal (IMC)}

Verificou-se existir diferença estatisticamente significativa entre os sexos nos valores médios de IMC $(\mathrm{p}<0,001)$, sendo o IMC inferior nos homens $(28,7)$ em relação às mulheres $(29,8)$.

Registou-se que 16,1\% dos diabéticos de tipo 2 tinha valores de IMC acima dos recomendados como objectivo de tratamento, sendo $15,6 \%$ homens e $16,5 \% \mathrm{mu}-$ lheres. Foi no grupo etário 80 e + anos que os objectivos de tratamento foram mais bem cumpridos, pois em $33,4 \%$ dos diabéticos o IMC era inferior a 25; pelo contrário, no grupo 45-54 anos registou-se o IMC mais elevado, com apenas $9,8 \%$ dos indivíduos com IMC $<25$.

Verificou-se haver diferenças estatisticamente significativas nos homens, nas mulheres e no total de ambos os sexos em relação à percentagem de diabéticos com IMC $<25$, por grupo etário $(\mathrm{p}<0,001)$. Para o grupo etário dos 25-34 anos verificou-se diferença estatisticamente significativa ( $\mathrm{p}=0,036)$.

\section{Proteinúria}

Verificou-se a existência de proteinúria em 409 (21,7\%) diabéticos.

Verificou-se haver diferença estatisticamente significativa no sexo masculino ( $\mathrm{p}=0,038)$, no sexo feminino $(p=0,045)$ e no total de ambos os sexos $(p=0,005)$ em relação à distribuição da proteinúria nos diabéticos de tipo 2 entre os vários grupos etários.

\section{Tensão arterial}

Verificou-se que apenas $16,9 \%$ dos diabéticos ( $\mathrm{N}=4.356$ ) de tipo 2 cumpriam as recomendações de tratamento (simultaneamente $<130$ e 80 ), sendo os homens $16,1 \%$ e as mulheres $17,6 \%$.

Os objectivos de tratamento foram mais bem conseguidos no grupo 25-34 anos, em ambos os sexos, respectivamente, com $45,5 \%$ nos homens e $42,9 \%$ das mulheres.

Verificou-se diferença estatisticamente significativa nos homens, nas mulheres e no total de ambos os sexos no que respeita à percentagem de diabéticos que cumpriam as recomendações relacionadas com a TA. O grupo etário dos 65 aos 74 anos apresentou diferença estatisticamente significativa $(\mathrm{p}=0,023)$.

\section{Complicações da diabetes}

Foram identificadas complicações da diabetes em 816 (18,7\%) diabéticos de tipo 2.

\section{Retinopatia diabética}

Verificou-se que $356(11,4 \%)$ diabéticos de tipo 2 tinham retinopatia diabética.

A frequência da retinopatia foi mais elevada no grupo etário $65-74$ anos (28,1\%), sendo $11,6 \%$ nos homens e $16,5 \%$ nas mulheres. Verificou-se haver diferença estatisticamente significativa nas mulheres e no total de ambos os sexos no que respeita à percentagem de diabéticos tipo 2 com retinopatia (Quadro VIII). No grupo etário dos 65-74 anos verificou-se diferença estatisticamente significativa $(\mathrm{p}=0,023)$.

\section{Cegueira Total}

Verificou-se que $17(0,4 \%)$ diabéticos de tipo 2 apresentavam cegueira total, sendo a percentagem mais elevada (0,6 \%) no grupo 65-74 anos.

\section{Hemodiálise}

Verificou-se que apenas 9 diabéticos $(0,4 \%)$ de tipo 2 se encontravam a fazer hemodiálise em 01/01/05.

\section{Diminuição grave da acuidade visual, não corrigível}

( $<1 / 10$ no melhor olho, cegueira legal)

Verificou-se que 79 (3,8\%) diabéticos de tipo 2 tinham, em 01/01/05, diminuição grave da acuidade visual, não corrigível, tendo a percentagem mais elevada $6,2 \%$, ocorrido no grupo $80 \mathrm{e}+$ anos.

Existe diferença estatisticamente significativa nos homens e no total de ambos os sexos no que respeita à 


\begin{tabular}{|c|c|c|c|c|}
\hline \multicolumn{5}{|c|}{$\begin{array}{l}\text { QUADRO VIII. Percentagem de diabéticos tipo 2, com } \\
\text { retinopatia diabética diagnosticada até 01/01/05, por } \\
\text { grupo etário e sexo }\end{array}$} \\
\hline Grupo etário & $\begin{array}{c}\text { Homens } \\
\%\end{array}$ & $\begin{array}{c}\text { Mulheres } \\
\%\end{array}$ & $\begin{array}{c}\mathrm{H}+\mathrm{M} \\
\%\end{array}$ & $P$ \\
\hline $25-34$ & $\begin{array}{c}0 \\
(9)\end{array}$ & $\begin{array}{c}0 \\
(4)\end{array}$ & $\begin{array}{c}0 \\
(13)\end{array}$ & - \\
\hline $35-44$ & $\begin{array}{c}7,8 \\
(64)\end{array}$ & $\begin{array}{c}4,1 \\
(49)\end{array}$ & $\begin{array}{c}6,2 \\
(113)\end{array}$ & $0,697^{a}$ \\
\hline $45-54$ & $\begin{array}{c}6,1 \\
(230)\end{array}$ & $\begin{array}{l}10,9 \\
(20)\end{array}$ & $\begin{array}{c}8,4 \\
(434)\end{array}$ & $0,081^{a}$ \\
\hline $55-64$ & $\begin{array}{c}10,8 \\
(389)\end{array}$ & $\begin{array}{c}9,3 \\
(409)\end{array}$ & $\begin{array}{c}10,0 \\
(798)\end{array}$ & $0,482^{\mathrm{a}}$ \\
\hline $65-74$ & $\begin{array}{c}11,6 \\
(493)\end{array}$ & $\begin{array}{c}16,5 \\
(583)\end{array}$ & $\begin{array}{c}14,2 \\
(1076)\end{array}$ & $0,023^{a}$ \\
\hline $75-79$ & $\begin{array}{c}9,6 \\
(156)\end{array}$ & $\begin{array}{c}16,4 \\
(220)\end{array}$ & $\begin{array}{c}13,6 \\
(376)\end{array}$ & $0,067^{a}$ \\
\hline 80 e + & $\begin{array}{c}10,4 \\
(125)\end{array}$ & $\begin{array}{c}8,2 \\
(194)\end{array}$ & $\begin{array}{c}9,1 \\
(319)\end{array}$ & $0,553^{\mathrm{a}}$ \\
\hline Total & $\begin{array}{c}10,0 \\
(1466)\end{array}$ & $\begin{array}{c}12,7 \\
(1660)\end{array}$ & $\begin{array}{c}22,7 \\
(3126)\end{array}$ & $0,018^{a}$ \\
\hline$P$ & $0,327^{b}$ & $0,001^{b}$ & $0,001^{b}$ & \\
\hline
\end{tabular}

(..) $\mathrm{n}^{\circ}$ de indivíduos com resposta válida; ateste Exacto de Fisher; ${ }^{\text {bteste de }} \chi^{2}$ de Pearson; sem informação: 28,2\% (1.230)

percentagem de diabéticos tipo 2 com diminuição grave da acuidade visual.

\section{Transplante renal}

Foi apenas identificado um diabético de tipo $2(0,02 \%)$, do sexo masculino, no grupo etário 65-74 anos, sujeito a transplante renal no passado $(\mathrm{N}-4.268$; $\mathrm{H}-1.0983$; M-2.267).

\section{Acidente Vascular Cerebral (AVC)}

Verificou-se que 309 (14,7\%) diabéticos de tipo 2 tinham antecedentes de AVC, tendo a percentagem mais elevada (25,7\%) ocorrido no grupo 80 e + anos.

Existe diferença estatisticamente significativa nos homens, nas mulheres e no total de ambos os sexos no que respeita à percentagem de diabéticos tipo $2 \mathrm{com}$ antecedentes de AVC.

\section{Enfarte Agudo do Miocárdio (EAM)}

Verificou-se que $173(8,5 \%)$ diabéticos de tipo 2 tinham antecedentes de EAM, tendo a percentagem mais elevada $(10,7 \%)$ ocorrido no grupo $80 \mathrm{e}+$ anos.

Verificou-se haver diferença estatisticamente significativa, no sexo masculino, no sexo feminino e no total de ambos os sexos em relação à percentagem de diabéticos tipo 2 com EAM diagnosticado. Os grupos etários dos 45-74 anos e 80 e mais anos apresentaram diferenças significativas, sendo as percentagens de diabéticos tipo 2 com EAM superiores nos homens.

\section{Bypass arterial periférico}

Verificou-se que 11 (0,5\%) diabéticos de tipo 2 tinham antecedentes de bypass arterial periférico.

\section{Angioplastia ou bypass coronário}

Verificou-se que $85(4,1 \%)$ diabéticos de tipo 2 tinham antecedentes de angioplastia ou de bypass coronário, tendo a percentagem mais elevada $(5,5 \%)$ ocorrido no grupo 65-74 anos.

Verificou-se haver diferença estatisticamente significativa, no sexo masculino e no total de ambos os sexos, em relação à percentagem de diabéticos tipo 2 com angioplastia ou bypass coronário. Os grupos etários 55 -64, 65-74 anos e 80 e + anos apresentaram diferenças significativas, sendo as percentagens de diabéticos tipo 2 com angioplastia ou bypass coronário superiores nos homens.

\section{Amputação}

Verificou-se que $40(0,9 \%)$ diabéticos de tipo 2 tinham sofrido, em 01/01/05, algum tipo de amputação. Dado o pequeno número de casos identificados, os dados não foram desagregados por grupo etário nem sexo.

\section{Dos dedos ou pés}

Verificou-se que $21(0,5 \%)$ diabéticos de tipo 2 tinham sofrido, em 01/01/05, amputação dos dedos ou dos pés.

\section{Perna}

Verificou-se que $12(0,3 \%)$ diabéticos de tipo 2 tinham sofrido, em 01/01/05, amputação pela perna.

\section{Coxa}

Verificou-se que $7(0,2 \%)$ diabéticos de tipo 2 tinham sofrido, em 01/01/05, amputação pela coxa.

No Quadro IX pode ver-se o quadro resumo das 


\begin{tabular}{|c|c|c|}
\hline \multicolumn{3}{|c|}{$\begin{array}{l}\text { QUADRO IX. Percentagem de complicações da diabetes } \\
\text { e de intervenções terapêuticas identificadas nos } \\
\text { diabéticos de tipo 2, por ordem decrescente. }\end{array}$} \\
\hline \multicolumn{2}{|l|}{ Complicações } & $\begin{array}{c}\% \text { de } \\
\text { diabéticos }\end{array}$ \\
\hline \multicolumn{2}{|l|}{ Retinopatia } & 11,4 \\
\hline \multicolumn{2}{|c|}{ Acidente vascular cerebral (AVC) } & 7,3 \\
\hline \multicolumn{2}{|c|}{ Enfarte Agudo do Miocárdio (EAM) } & 4,1 \\
\hline \multicolumn{2}{|c|}{$\begin{array}{l}\text { Diminuição grave da acuidade visual, } \\
\text { não corrigível }(<1 / 10 \text { no melhor olho) }\end{array}$} & 1,9 \\
\hline \multirow{4}{*}{ Amputações } & $\begin{array}{l}\text { Pelo menos uma amputação, } \\
\text { a qualquer nível }\end{array}$ & 0,9 \\
\hline & Dos dedos ou pés & 0,5 \\
\hline & Da perna & 0,3 \\
\hline & Da coxa & 0,2 \\
\hline \multicolumn{2}{|c|}{ Cegueira total } & 0,4 \\
\hline \multicolumn{3}{|c|}{ Intervenções terapêuticas } \\
\hline \multicolumn{2}{|c|}{ Angioplastia ou bypass coronário } & 2,0 \\
\hline \multicolumn{2}{|c|}{ Bypass arterial periférico } & 0,3 \\
\hline \multicolumn{2}{|c|}{ Hemodiálise } & 0,2 \\
\hline \multicolumn{2}{|c|}{ Transplante renal } & 0,0 \\
\hline
\end{tabular}

percentagens de complicações da diabetes ou de intervenções terapêuticas identificadas nos diabéticos de tipo 2.

\section{DISCUSSÃO}

Apesar do grande volume de dados recebidos durante este estudo, os resultados obtidos poderão não corresponder exactamente à realidade do país. De facto, os médicos participantes neste estudo pertencem à Rede Médicos-Sentinela, e, só por esta razão, poderão ter listas de utentes com características diferentes das de outros colegas, seja no número de diabéticos e nas respectivas características, seja nos padrões de prescrição utilizados.

Ora, considerando que o trabalho na rede é voluntário e que dela se excluem médicos de Medicina Geral e Familiar (MGF) que não trabalham em Centros de Saúde, facilmente se aceitará que os médicos de MGF que participam na Rede Médicos-Sentinela poderão não ser representativos dos médicos de MGF espalhados por todos o país.

Por outro lado, sabe-se que alguns médicos, pelo facto de terem listas de utentes com uma elevada propor- ção de diabéticos, não terão querido participar no estudo, em virtude do enorme acréscimo de trabalho que tal participação acarretaria; e que outros médicos, por razões várias, poderão não ter enviado todos os dados sobre os seus utentes diabéticos; e que as listas de utentes dos médicos participantes poderão não estar completamente actualizadas, e incluir, ainda, utentes que, de facto, já não irão mais à consulta daquele médico, por razões várias, como morte, emigração, mudança de residência ou outros motivos. Ora, apesar de não ser possível estimar as percentagens referentes a cada um destes factores, sabe-se que, se forem elevadas, poderão constituir um viés a ter em conta e originar, por exemplo, uma sub-estimação da taxa de prevalência da diabetes; assim, os resultados agora apresentados deverão sempre ser considerados como valores mínimos.

Verificou-se que a quase totalidade $(95,3 \%)$ da coorte de diabéticos estudada era constituída por diabéticos de tipo 2 e que apenas $3,5 \%$ era de tipo 1 ; os restantes $1,2 \%$ eram diabetes de «outro tipo» ou «gestacionais.

A taxa de prevalência de diabetes de tipo 1 foi estimada em $0,1 \%$ para ambos os sexos. Este valor, ajustado à população portuguesa, permitiu estimar em 15.003 o número de diabéticos de tipo 1 na população portuguesa.

A taxa de prevalência da diabetes de tipo 2 foi estimada em 5,4\% para ambos os sexos e separadamente para homens e mulheres. Este valor, ajustado à população portuguesa, permitiu estimar em 414.214 o número de diabéticos de tipo 2 na população portuguesa, sendo 194.855 nos homens e 219.359 nas mulheres. Estas estimativas de prevalência poderão parecer subavaliadas quando comparadas com os resultados das estimativas obtidas através do $4^{\circ}$ Inquérito Nacional de Saúde. ${ }^{13}$ De facto, o INS estima em $6,5 \%$ a prevalência de diabetes na população portuguesa. Saliente-se que este valor resulta de informação auto-declarada pelos indivíduos, sem qualquer confirmação médica. Só por esta razão, pode-se admitir que haja aqui um viés de informação, embora não se saiba qual o seu sentido, i.e, tão plausível parece a sobre como a sub auto-declaração de diabetes. De facto, alguns indivíduos com diabetes poderão não a ter declarado, por várias razões; e outros poderão ter declarado ser diabéticos sem que, de facto, lhes tenha sido colocado esse diagnóstico. Pelo contrário, os resultados obtidos pela Rede Médicos-Sentinela foram confirmados pelo médico, embora se 
admita que poderão estar sub-avaliados, como já foi referido.

\section{Diabéticos de tipo 2 \\ Terapêutica prescrita}

As terapêuticas prescritas foram, por ordem decrescente de número de utentes, os antidiabéticos orais $(87,4 \%)$, os antihipertensores $(75,5 \%)$, a dieta/exercício físico $(62,8 \%)$, os antidislipidémicos $(56,3 \%)$, a terapêutica antitrombótica $(37,1 \%)$, a terapêutica para prevenção/tratamento de nefropatia $(30,0 \%)$ e a insulina $(9,3 \%)$. Pode, assim, concluir-se, haver algum tipo de co-morbilidade associada à diabetes, nomeadamente hipertensão arterial, diagnosticada em cerca de $2 / 3$ dos diabéticos e dislipidémia, em cerca de pouco mais de metade. Saliente-se que a dieta e o exercício só foram registados como terapêutica em $62,8 \%$ dos casos, o que provavelmente corresponde a um erro de preenchimento ou de interpretação, já que é consensual que todos os diabéticos devem seguir um programa de hábitos alimentares saudáveis e de exercício físico.,20

\section{Hábitos tabágicos}

Nesta coorte verificou-se que a maior parte dos diabéticos (93\%) não tinha hábito tabágicos; mas, nos que os tinham, a proporção de homens foi significativamente superior à das mulheres. Comparando os hábitos tabágicos dos diabéticos estudados com os da população portuguesa, verificou-se que são menos acentuados na coorte de diabéticos. De facto, a proporção de diabéticos fumadores é cerca de 4 vezes inferior à que foi estimada para a população portuguesa e, em ambas as populações, a proporção de homens fumadores foi maior do que a das mulheres, i.e., cerca de 8 vezes mais na coorte de diabéticos e cerca de 3 vezes mais na população portuguesa.

\section{Hábitos alcoólicos}

Verificou-se que cerca de metade $(50,2 \%)$ dos diabéticos não tinha hábitos alcoólicos; mas, nos que «bebiam excessivamente» (6,1\%), a proporção de homens foi significativamente superior à das mulheres.

Comparando os hábitos alcoólicos dos diabéticos estudados com os da população portuguesa não parece haver grande diferença, quando se considera o Continente e a RAA, mas exceptuando a RAM. De facto, a proporção de diabéticos com hábitos alcoólicos (49,8\%) foi ligeiramente inferior à da população portuguesa do Continente $(53,8 \%)$, muito semelhante à da RAA $(48,8 \%)$ e superior à da RAM $(31,5 \%)$.

\section{Consulta de oftalmologia}

Verificou-se que quase metade dos diabéticos $(48,7 \%)$ tinha ido à consulta de oftalmologia no ano de 2004, em proporções semelhantes nos dois sexos, o que parece estar longe do objectivo proposto, que é uma consulta anual. ${ }^{20}$ Este facto, só por si, pode ter originado uma sub-estimativa da prevalência da retinopatia diabética.

\section{Cumprimento dos objectivos explicitados nas Recomendações de $\mathbf{2 0 0 6}$ do Grupo de Estudos da Diabetes da APMCG ${ }^{20}$}

Em relação ao cumprimento dos objectivos explicitados nas Recomendações de 2006 do Grupo de Estudos da Diabetes da APMCG na diabetes tipo 2 para a prática clínica diária em Cuidados de Saúde Primários, foi possível concluir, através de alguns indicadores, que o que mais frequentemente esteve de acordo com os valores recomendados, foi o valor dos triglicéridos (60,2\%); pelo contrário, o índice de massa corporal foi o que menos frequentemente esteve conforme aqueles valores ( $16,1 \%$ dos casos). A HbAlc, o colesterol total e o colesterol-HDL tinham valores recomendados em cerca de metade dos casos e o colesterol-LDL e a tensão arterial em menos de $30 \%$ dos casos.

\section{Complicações da diabetes}

A interpretação destes resultados deve ser muito cuidadosa, uma vez que o número de casos é reduzido.

Foram identificadas complicações da diabetes em $18,7 \%$ dos diabéticos de tipo 2 , tendo sido a partir dos 65 anos que se verificaram as percentagens mais elevadas de cada uma delas.

A retinopatia diabética foi a complicação da diabetes mais frequentemente identificada $(11,4 \%)$. Ainda assim, é importante ter em conta que este valor pode estar sub-avaliado, uma vez que apenas metade dos utentes tinha ido à consulta de oftalmologia no ano anterior. Desta forma, podemos supor que alguns dos diabéticos que não foram a essa consulta poderiam também ter o diagnóstico de retinopatia diabética, caso aí tivessem ido. 
A diminuição grave da acuidade visual pode estar, aqui, sobreavaliada, uma vez que diminui com a idade, independentemente do indivíduo ser ou não diabético.

Espera-se que os resultados deste relatório possam contribuir para o melhor conhecimento da diabetes em Portugal. Aguarda-se que a $3^{\text {a }}$ fase deste estudo seja finalizada para se estimarem taxas de mortalidade e de incidência das várias complicações.

\section{REFERÊNCIAS BIBLIOGRÁFICAS}

1. American Diabetes Association. Standards of medical care in diabetes - 2006. Diabetes Care 2006 Jan; 29 Suppl 1: S4-42.

2. Williams G, Pickup JC. Handbook of Diabetes. 3rd ed. Malden, Mass.: Blackwell; 2004.

3. Internacional Diabetes Federation. e-Atlas. Disponível em: http:// www.eatlas.idf.org [acedido em 07/11/2008].

4. Ford ES, Williamson DF, Liu S. Weight change and diabetes incidence: findings from a national cohort of US adults. Am J Epidemiol 1997 Aug $1 ; 146$ (3): 214-22.

5. Björntorp P. Obesity. Lancet 1997 Aug 9; 350 (9075): 423-6.

6. National Center for Chronic Disease Prevention and Health Promotion. Physical activity and health: a report of the Surgeon General. Atlanta: Centre for Disease Control and Prevention; 1996.

7. Kelly DT. Our future society: a global challenge. Circulation 1997; 95 : 2459- 64.

8. OMS.What is Diabetes? Disponível em: http://www.who.int/mediacentre/factsheets/fs312/en/index.html [acedido em 02/07/2008].

9. American Diabetes Association. Economic consequences of diabetes mellitus in the U.S. in 1997. Diabetes Care 1998 Feb; 21 (2): 296-309.

10. Sistema de Saúde Português. Disponível em: http://193.136.116.5/saboga/prosaude/eumahp/profile\%20D.doc [acedido em 01/07/2008].

11. Programa Nacional de Prevenção e Controlo da Diabetes. Disponível em: http://www.min-saude.pt/NR/rdonlyres/92EE1644-43DC-4A1597FF-87FDC3BD7B00/0/i009220.pdf [acedido em 02/07/2008].

12. Divisão de Epidemiologia e Bioestatística da Direcção-Geral da Saúde. Epidemiologia da diabetes. Lisboa: Direcção-Geral da Saúde; 1994.

13. $4^{\circ}$ Inquérito Nacional de Saúde. Disponível em: http://www.insa.pt/sites/INSA/Portugues/ComInf/Noticias/Paginas/Indicadoresadicionais4INS.aspx [acedido em 07/12/2008].
14. Associação Protectora dos Diabéticos de Portugal. Disponível em: http://www.apdp.pt/diabetes.asp [acedido em 03/07/2008].

15. American Diabetes Association. Standards of medical care in diabetes - 2006. Diabetes Care 2006 Jan; 29 Suppl 1: S4-42.

16. OMS. International guide for monitoring alcohol consumption and related harm. Disponível em: http://whqlibdoc.who.int/hq/2000/WHO_ MSD_MSB_00.4.pdf [acedido em 03/07/2008].

17. General Practice notebook. Disponível em: http://www.gpnotebook. co.uk/simplepage.cfm?ID=x20030114211535665170 [acedido em 03/07/2008].

18. OMS. Body Mass Index. Disponível em: http://www.who.int/bmi/index.jsp? introPage=intro_3.html17 [acedido em 01/07/2008].

19. Instituto Nacional de Estatística. Estimativa da população portuguesa residente em 2006. Disponível em: http://www.ine.pt/xportal/xmain? xpid=INE\&xpgid=ine_publicacoes\&PUBLICACOESpub_boui= 16223823\&PUBLICACOESmodo=2 [acedido em 01/07/2008].

20. Associação Portuguesa dos Médicos de Clínica Geral. Recomendações 2006. Disponível em: http://www.apmcg.pt/PageGen.aspx?WMCM_Paginald=27436 [acedido em 03/07/2008].

21. SPSS $15^{\circledR}$ for Windows. SPSS inc. 2006

\section{AGRADECIMENTOS}

Ao Dr. José Manuel Boavida, Director Clínico daAssociação Protectora dos Diabéticos de Portugal, pelo contributo dado na fase transversal deste estudo.

Aos Médicos de Família da Rede Médicos-Sentinela que, duma forma eficaz e determinada, possibilitaram a realização deste estudo.

\section{ENDEREÇO PARA CORRESPONDÊNCIA}

Isabel Marinho Falcão

Direcção-Geral da Saúde

Unidade de Apoio às Emergências de Saúde Pública

Alameda D. Afonso Henriques, 45

1049-005 Lisboa

E-mail: ifalcao@dgs.pt

Tel: 218430628

Recebido em 22/08/08

Aceite para publicação em 12/12/08 


\section{ABSTRACT}

Objectives: To characterize a cohort of diabetics by sex and age; to estimate the prevalence of diabetes type 1 and type 2; to characterize the prescribed therapeutic to type 2 diabetic patients and their consumption of tobacco and alcohol, ophthalmology consultation review and some biometrical and biochemical data; and to estimate prevalence of type 2 diabetes complications.

Type of study: A one year transversal phase from a 3 years follow up study.

Place: Population related to several Health Centres.

Population: A cohort of 4583 diabetic patients from 66 general practitioners lists collaborating with the portuguese sentinel network.

Methods: Survey of general practitioners their diabetic patients. Data have been analysed in the Department of Epidemiology of the Portuguese National Institute of Health Dr. Ricardo Jorge.

Results and conclusions: The prevalence of diabetes type 1 and type 2 have been estimated in $0,1 \%$ and 5,4\%, respectively. Hypertension has been the most frequent therapeutic prescribed associated to diabetes, for 3265 (75,5\%) cases. One need to be careful concerning interpretation of the results related with the diabetes complications, considering the small number of cases. Diabetes complications have been identified for $816(18,7 \%)$ type 2 diabetic patients, as the retinopathy was the most frequent ( $356 ; 11,4 \%$ cases).

As about half of the diabetic patients had been consulted for ophthalmology during the previous year (1790; 48\%), one may underestimate the prevalence of this complication.

Although the big amount of data, it is known that several factors could have biased the results; then, they should be considerate as minimal.

Keywords: Diabetes; Prevalence; Complications; Therapeutic; Portuguese Sentinel Network. 